\title{
Estrategia de intervención para optimizar la comunicación con padres de familia en Bogotá
}

\author{
Intervention strategy for optimizing the communication with parents in \\ Bogota
}

\author{
Carlos Escobar (0000-0003-3107-5315) , Judith Yangali (0000-0003-0302-5839)² \\ ${ }^{1}$ Colegio General Gustavo Rojas Pinilla IED \\ ${ }^{2}$ South American Center for Education and Research in Public Health - Escuela de \\ Posgrado de la Universidad Norbert Wiener
}

\begin{abstract}
Resumen
En las instituciones educativas, la comunicación es un elemento vital que requiere de una dinámica funcional acorde con necesidades de la población y el número de estudiantes. Buscando herramientas que reemplacen el canal tradicional y ayuden a toda la comunicación escolar, se presenta a InCSEI, como estrategia de intervención dentro de una investigación aplicada, así como implementar la TIC, la cual propondría la manera de potenciar el trabajo. El objetivo de este artículo, entonces, es analizar las TIC en escuelas como herramienta de comunicación para identificar el grado de mejora en la optimización de la comunicación. La intervención fue realizada en el Colegio General Gustavo Rojas Pinilla IED, en la ciudad de Bogotá, en el año 2019. La investigación responde a un enfoque cuantitativo de diseño experimental con subdiseño cuasi experimental. En el artículo se realiza un análisis que comprende la caracterización y el análisis unidimensional de las variables; y, a su vez, un análisis de contraste de variable. Se concluye que se evidencia la percepción de los padres respecto a la fluidez y eficacia de la comunicación con el uso de la herramienta, así como la satisfacción de estos en el mejoramiento en la comunicación institucional.
\end{abstract}

Palabras clave: Comunicación, canales, ecología cultural, prosumidores software, InCSEI. 


\begin{abstract}
In educational institutions, communication is a vital element, which requires a functional dynamic according to the needs of the population and number of students. Looking for tools to replace the traditional channel and help the whole school communication, InCSEI is presented as an intervention strategy, within an applied research, implementing ICT, which would propose the way to enhance the work. As an objective: To analyze ICT in schools as a communication tool to identify the degree of improvement for the optimization of communication, in the General Gustavo Rojas Pinilla School IED, in the city of Bogota, year 2019. The research responds to a quantitative approach of experimental design with quasi-experimental sub-design. An intervention was carried out and an analysis was made comprising the characterization and unidimensional analysis of the variables, as well as an analysis of variable contrast. It is concluded that the parents' perception of the fluidity and effectiveness of communication with the use of the tool and their satisfaction in the improvement of institutional communication was evidenced.
\end{abstract}

Keywords: Communication, channels, cultural ecology, software prosumers, InCSEI.

\section{Introducción}

La comunicación es uno de los elementos que nos diferencian de los demás animales dentro de la escala evolutiva. Es una cualidad racional y emocional específica de los seres humanos, que surge de la necesidad de ponerse en contacto con los demás y que permite el intercambio de ideas (Fonseca, Correa, Pineda y Lemus, 2011).

El concepto de comunicación se ha ido transformando y, con el uso de las nuevas tecnologías de la información y la comunicación (TIC), las formas de comunicación han continuado transformándose, lo cual hace que las personas tengan mayor acceso a la información y se pueda acortar tiempos y errores en la trasmisión del mensaje. Esta línea de la comu- nicación propone los conceptos glocales, prosumidores y la ecología cultural (Islas, 2008), lo cual está asociado al paradigma de las bases de los componentes que inician con el paradigma de Harold Dwight Lasswell (1948), quien lo propone en su escrito Estructura y función de la comunicación en la sociedad. Lasswell parte de las estructuras básicas de la comunicación propuesta por Jürgen Habermas, quien renueva la tesis estructural funcionalista y sistémica de Parsons (Habermas, 1981), y el modelo sintético de la comunicación de Niklas Luhmann descrito por Pignuoli (2013), quien lo describe como operación de los sistemas sociales y la autopoiesis comunicativa, los cuales se visualizan la sociedad como un sistema vivo que se regula propuesto. 
Desde la propuesta Cavazza (2016), se definen las acciones que gobiernan la conducta de los medios que están en las redes, como la propaganda, el manejo de los contenidos, la intervención en la información, el uso de email, el debate en los temas propuestos, el trabajo colaborativo, y la creación de tejidos virtuales. Cuando esta dinámica comunicativa es incluida en las instituciones educativas se genera un sistema masivo de comunicación científica y tecnológica a través de la plataforma GLOCAL (UNESCO, 2019).

Las investigaciones de Garza (2017) ponen en evidencia los prejuicios y el analfabetismo frente al beneficio que ofrecen los medios sociales para, a través de las redes, descubrir el mundo. Se pretende reconocer la necesidad de que los docentes tengan una constante comunicación con los padres, lo cual les permitiría conocer los avances y dificultades de sus hijos, frente a cualquier situación de riesgo o de rendimiento académico.

La propuesta de mejoramiento de la comunicación en instituciones educativas del Colegio General Gustavo Rojas Pinilla IED, o en cualquier institución educativa, surge de la necesidad de realimentación de la información en forma bidireccional. Se ha logrado la creación de una herramienta-InCSEI: Internal Communication System for Education Institutions- virtual de comunicación sincrónica y asincrónica.

A su vez, se han realizado investigaciones diferentes áreas específicas de comunicación a nivel individual, diádico, grupal y organizacional (Keyton, 2017); con lo cual se ha abierto un espacio de aplicación a múltiples sectores empresariales y, entre ellas, a las instituciones educativas.
La utilización de herramientas tecnológicas es un desafío que concluye con la apertura de la comunicación al internet, flexibiliza y amplia los límites de la organización (Barbu y Şomăcescu, 2016). La aplicación adecuada de las nuevas tecnologías conduce no solamente a la redefinición del concepto de comunicación, sino también a la reestructuración de la organización misma, pues se hace más ágil al aceptar e incorporar los nuevos cambios. Cuando los sistemas de comunicación cambian, esta debe ser asertiva, consciente y debe permitir el acercamiento entre los actores educativos. Con esto, se facilita la depreciación de las brechas comunicativas existentes, se permite que estudiantes y padres sean los actores activos en el proceso de formación, y se optimizan las relaciones personales que permitan un aprendizaje significativo (Jiménez, 2017). La acción comunicativa adecuada entre los padres y la escuela incide en la formación de los estudiantes (Castro, 2015).

El objetivo es lograr un análisis de la comunicación en instituciones educativas internas, el cual se genera entre docentes y directivos en una institución educativa, teniendo como marco referencial el mejoramiento de la gestión educativa (Papic, 2016). La adaptación del contexto propio de cada institución requiere de códigos propios que pueden implementarse desde la TIC, como un nuevo paradigma de comunicación. Este paradigma es conocido como "Era de convergencia", la cual es la acción comunicativa que demanda la sociedad, y que permite, además, reconocer sus falencias en los sistemas de comunicación (Hasbún, 2015) . Con esto se reconocen los cambios en las sociedades donde la 
aplicación de elementos tecnológicos modifica el sistema de comunicación y adapta las sociedades de información y conocimiento.

Las tecnologías de la información y la comunicación "son aquellos dispositivos tecnológicos cuyos usos incluyen la creación, modificación, almacenamiento y recuperación de información, contribuyendo a la generación de conocimiento"(Martinez- Alvarez et al., 2021, p. 5595). Las tecnologías de la información y la comunicación "influyen de manera significativa tanto para refrescar, como para cambiar la enseñanza y mejorar el aprendizaje" (Yangali, Arispe y Arboleda, 2020, p. 156).

Los sistemas de comunicación virtual de esta investigación se centran el Modelo de comunicación digital para ambientes glocales (Islas, 2008), el cual es el paradigma de esta investigación. La comunicación es un sistema de códigos que permiten la interacción entre dos o más individuos; cada uno de los cuales en un momento es catalogado como emisor y el otro como receptor dependiendo de la direccionalidad de la comunicación. La comunicación en educación es un campo poco abarcado desde la investigación, pero existen algunas perspectivas teóricas reconocidas, entre ellas como la teoría de Habermas (1981), que es una simbiosis de todas las influencias sociales y cognitivas de su época, entre las que se encuentran la crítica racionalista de la Escuela de Frankfurt, la sociología de Weber, centrada en las opiniones de la racionalidad y utilidad, la importancia del lenguaje del simbólico y la influencia del sujeto (Aguado, 2004). Esto hace que Habermas logre definir una tipo- logía de las acciones sociales, entre ellas la acción comunicativa, que se define por estar orientada a la comprensión de las ideas del otro y el logro de consenso. Laacción comunicativa se define como un elemento que propone reglas racionales intersubjetivas que hacen posible el entendimiento, teniendo en consideración que la sociedad se entiende como un sistema intersubjetivo de funcionalmente diferenciado por normas y significados (Aguado, 2004).

Niklas Luhmann (1980) se centra en las teorías sociológicas que determinan las organizaciones y procesos sociales del sujeto. Centra sus posiciones en la sociología estructural funcionalista de Talcott Parsons, la teoría general de Sistemas de Ludwig Von Bertalanffy y la Cibernética propuesta por Norbert Wiener (Aguado, 2004). Luhmann (1980) propuso el modelo sintético de la comunicación, que describe como operación de los sistemas sociales y la autopoiesis comunicativa, y que describen a la sociedad es un sistema vivo que se regula (Pignuoli, 2013).

Harold Dwight Lasswell (1948) propone en su escrito Estructura y función de la comunicación en la sociedad los elementos de una adecuada comunicación. En el artículo relaciona la estructura de la comunicación con su función y las necesidades sociales de comunicación. El paradigma de Lasswell logra de forma adecuada preguntarse por el significado de la comunicación, la cual surge de dar respuesta a preguntas como ¿Quién dice?, ¿qué?, ¿en qué canal?, ¿a quién?, y, ¿con qué efecto? (Lasswell, 1948). La comunicación verbal, que puede ser oral o escrita, está plasmada en la tabla 1 , en la cual se realiza un cuadro comparativo de estos dos medios. 


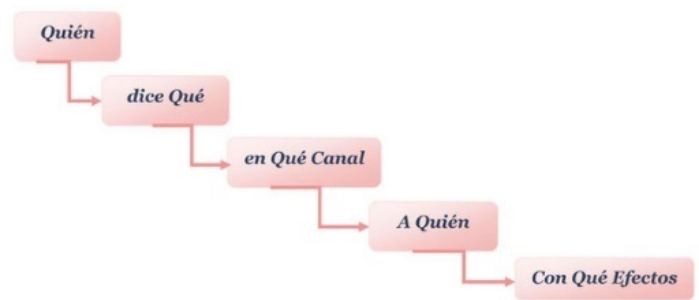

Figura 1: Representaciones del modelo Lasswel para comunicación (Comunicólogos, 2017)

El modelo de comunicación el de Lasswell es el método más aceptado, pues describe la relación comunicativa entre el emisor y el receptor a través de un canal definido. Para Lasswell (1948), el proceso comunicativo dentro de la sociedad tiene definidos cuatro componentes: la vigilancia del entorno, la correlación de los componentes de la sociedad, la transmisión del legado social y el entretenimiento. Este paradigma reproduce la comunicación como un proceso de transmisión lineal de un mensaje que parte del emisor hacia el receptor a través de un canal apropiado y con el código correspondientemente compartido por ambos agentes.

Los dispositivos digitales posibilitan nuevas formas de interactuar más allá de la simple recepción de información, para permitir el desarrollo del rol de prosumidor y de las posibilidades de interactuar a través de la internet (Islas, 2008).

Esta investigación centra su hipótesis en el mejoramiento de la comunicación en instituciones educativas desde el uso de herramientas TIC. Islas (2009) sostiene que la sociedad actual está ante la búsqueda de una ecología de la cultura, y propone el concepto de globalización, el cual define que el consumo en un específico tiempo y la comprensión de la realidad están ligadas a la influencia que ejercen los medios, en la mente y en el comportamiento de los sujetos.

El uso de la web permite a los individuos la creación y la posterior puesta a disposición de información que usan como canal los dispositivos o interfaces digitales, ya sean móviles como celulares y tabletas, o estacionarias con los computadores, desarrollados por los prosumidores. Como afirma Islas (2008), son ellos quienes proponen las condiciones necesarias para acceder a ambientes virtuales, transitando a una ecología cultural.

En un ambiente de ecologías culturales precedentes, se pone en marcha la nueva ecología cultural marcada por el fenómeno de la globalización, en la cual los dispositivos digitales posibilitan nuevas formas de interactuar más allá de la simple recepción de información, para permitir el desarrollo del rol de prosumidor y de las posibilidades de la ubicuidad en la cual la web pone a una persona, en cualquier momento y en cualquier lugar, a interactuar a través de ésta. 


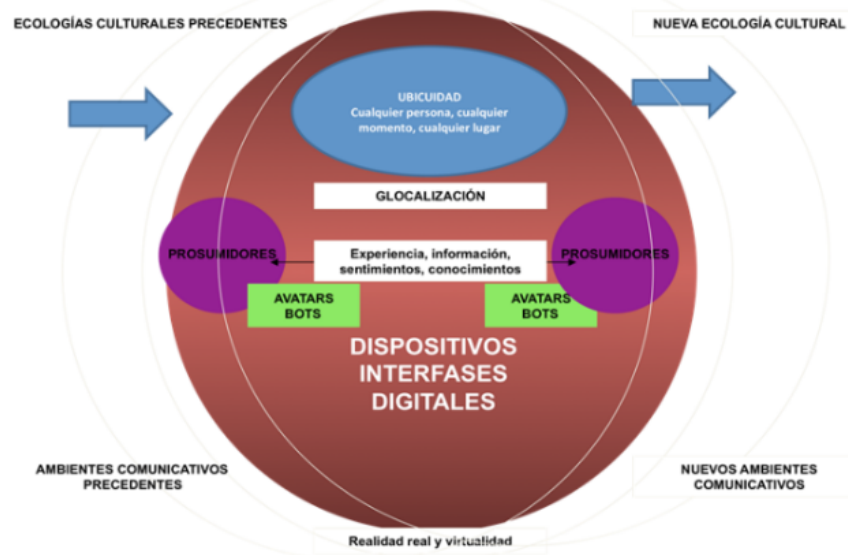

Figura 2: Representación del modelo de Octavio Islas (Islas, 2008)

\subsection{Estrategias de intervención para potenciar la comunica- ción}

El Colegio General Gustavo Rojas es una institución educativa institucional, ubicada en la ciudad de Bogotá DC. Se encuentra en la UPZ Castilla. Está activo desde el año 2008, cuenta con 3500 estudiantes y 150 docentes en dos jornadas. La necesidad de comunicación surge de la evaluación de acreditación en alta calidad del año 2016, se propone buscar o diseñar un sistema más ágil de para comunicarse. De ahí surge esta propuesta.

El software InCSEI es un sistema de comunicación en instituciones educativas orientado a instituciones educativas; cuenta con un sistema móvil de operación para los usuarios y una de plataforma web de gestión y generación de reportes para el área administrativa. Todo esto ha sido planteado para el apoyo y la optimización del flujo de información determinante para gestionar el desarrollo integral de los estudiantes (desde los procesos académicos hasta convivenciales) en las instituciones educativas. El estudio fue una propuesta creativa del docente, que es una característica trial, y que se comporta como inteligencia, habilidad o capacidad (Tunjo, Yangali, 2021).

Este sistema de comunicación web permitirá registrar información del proceso de enseñanza para ser transmitidas o notificadas a los actores que intervienen. De esta forma, se genera una completa gestión de la comunicación interna que se maneja en la institución educativa.

InCSEI, es la herramienta TIC utilizada. Es un software de comunicación para el mejoramiento de la comunicación en instituciones educativas. La implementación de la herramienta tecnológica buscó minimizar las brechas presentadas en el proceso de comunicación institucional, lo cual arrojó la evaluación institucional y el informe de gestión de calidad 2016 - 2017 . En este último se evidencia que los mecanismos son lentos y los procesos no están 


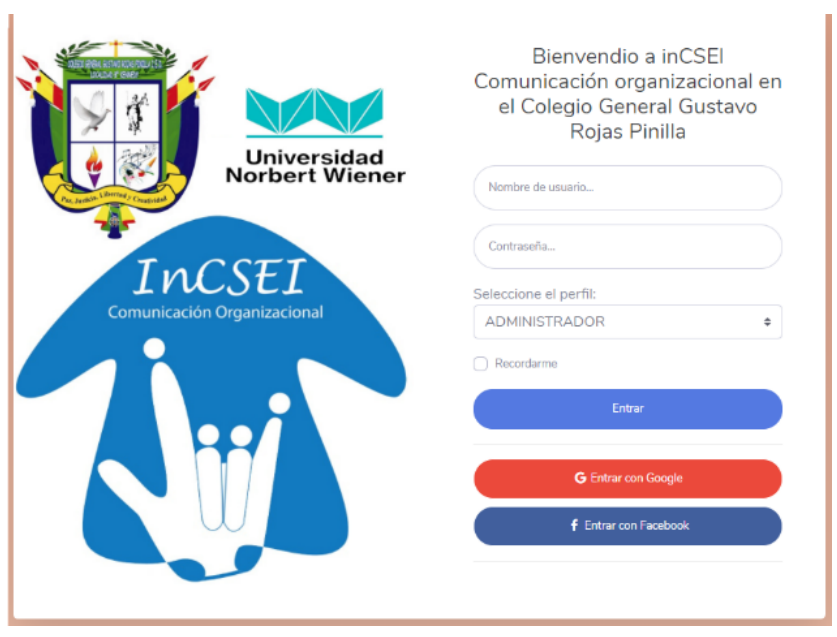

Figura 3: Presentación inicial del software InCSEI.

articulados, ni se contaba, asimismo, con una validación de la recepción de la información que ayuda a mejorar sustancialmente los procesos comunicacionales.

El uso del software InCSEI, como sistema de comunicación bidireccional, permite darles información a los padres, de forma sincrónica o asincrónica, de situaciones que se dan dentro de la institución educativa. Esto generan acciones preventivas o correctivas. Los resultados son validados a partir del juicio de expertos sobre la prueba. Se contextualiza al objeto de estudio, y se coteja la información que se analizó desde el uso de modelos estadísticos usando como referencia el marco teórico conceptual de comunicación en instituciones educativas y el uso de herramientas TIC en procesos de comunicación.

El estudio se ha desarrollado a través del análisis de cuatro dimensiones de las variables Implementación de Herramienta TIC (independiente) y Comunicación en instituciones educativas (dependiente). Estas permitieron recoger información va- liosa desde el pretest y el postest, con lo cual se logró hacer una clara descripción del alcance logrado con el uso de la herramienta TIC en la comunicación en instituciones educativas en el presente colegio.

\section{Metodología}

Este estudio correspondió a una investigación aplicada en la que profundiza el estudio de las variables como aporte al conocimiento de las mismas (Arispe et al., 2020). La investigación responde al enfoque cuantitativo, dado que utiliza un pretest y un postest, para la recolección de la información y su posterior análisis; asimismo, se contrastan los resultados para probar las hipótesis propuestas (Hernández, Fernández y Baptista, 2014).

El método de investigación fue hipotético deductivo, el diseño es experimental, de sub diseño cuasi experimental, de corte longitudinal y el nivel es explicativo. La técnica utilizada fue la encuesta y el 


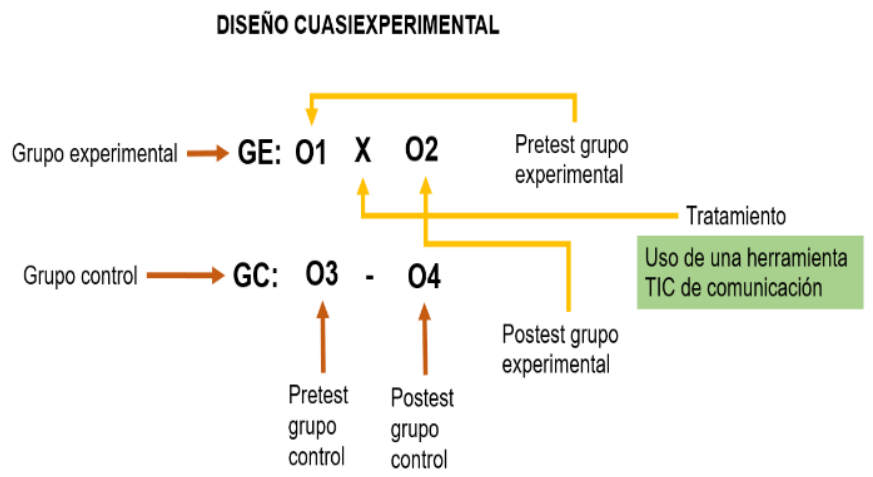

Figura 4: Diseño cuasiexperimental

instrumento fue el cuestionario.

La estrategia de intervención consistió en la inclusión de un sistema de comunicación que permite a los padres de familia el conocimiento de algunas acciones de los estudiantes y de la vida institucional, que le fortalecen el acercamiento al colegio. El software les permitió acceder a información de su hijo/a, conocer las modificaciones del calendario académico, actividades estudiantiles y recibir comunicación institucional a través de circulares. Se describe la estrategia con los encuentros realizados con el consejo directivo, consejo académico, equipo de gestión, rectoría, padres, docentes y estudiantes. Se registran los pantallazos del software InCSEI, reportes y una línea de tiempo de la comunicación docente estudiante, y registro fotográfico de los encuentros.

La técnica que se utilizó en esta tesis fue la encuesta a una muestra censal, la cual permitió la recolección de datos que proporcionan los individuos de una población, para identificar sus opiniones, apreciaciones, puntos de vista, actitudes, intereses o experiencias, entre otros aspec- tos. Todo esto se efectuó mediante la aplicación de cuestionarios, técnicamente diseñados para tal fin (Niño, 2011) . Esta técnica se basó en la realización de un sondeo con los padres de estudiantes, con quienes se validó el software. El procedimiento fue el siguiente: A un grupo se le aplica una encuesta, después se le administra el tratamiento y, finalmente, se le aplica una segunda encuesta posterior al tratamiento (Hernández, Fernández y Baptista, 2014).

Los datos fueron procesados y promediados teniendo en consideración las variables y los indicadores; para esto se realizaron dos pasos: la prueba de normalidad y el testeo de hipótesis a través de U Man Witney. La finalidad fue lograr el propósito de identificar el grado de mejoramiento de la implementación de herramientas TIC en la comunicación padres - docentes (Espinoza, 2014) . Esta investigación se desarrolló cumpliendo con autorizaciones necesarias para el tratamiento de la información.

Se solicitó la autorización al consejo directivo del Colegio General Gustavo Ro- 


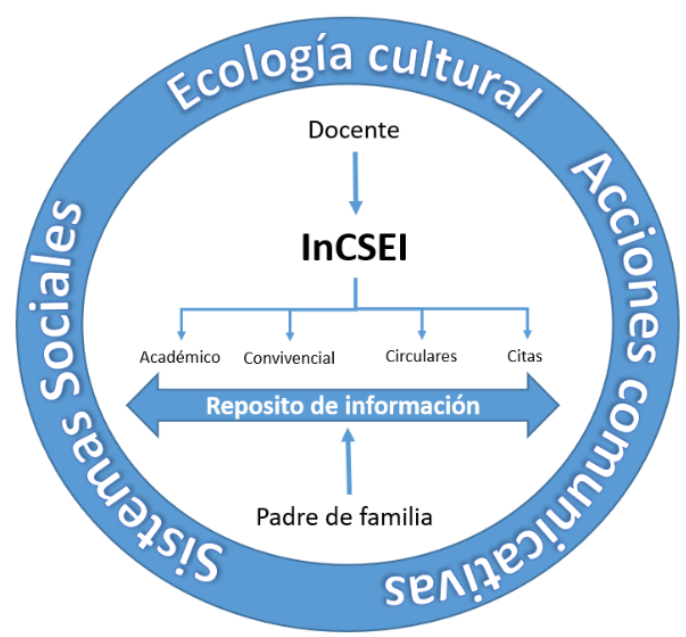

Figura 5: Diseño de la estrategia de intervención (Islas, 2008; Pignuoli, 2013)

Ficha técnica de las pruebas de entrada y salida

\begin{tabular}{lll}
\hline CRITERIOS & PRETEST & POSTEST \\
\hline Técnica: & Encuesta & Encuesta \\
Ámbito geográfico & Bogotá D.C & Bogotá D.C \\
Universo & Padres de familia & Padres de familia \\
\hline Población & 3200 padres & 3200 padres \\
$\mathrm{N}^{\circ}$ de la muestra & 105 padres & 105 padres \\
Valor representativo & 83 encuestas & 83 encuestas \\
Error: & $+/-0,5$ & $+/-0,5$ \\
Fecha de realización & Febrero 11 & Junio 10 \\
\hline Muestreo: & 18 padres & 18 padres \\
\hline
\end{tabular}

Fuente: diseño propio de la investigación

jas Pinilla IED, para acceder a la base de datos e iniciar la convocatoria a padres. A los convocados se les explicó el objetivo de la investigación y el objeto de estudio, y estos firmaron el consentimiento informado, cumpliendo con los compromisos del investigador, del manejo total de la reserva de la información que circule a través del software InCSEI, acorde con las normas nacionales e internacionales para el manejo de datos personales.

\section{Resultados}

Según plantean Berlanga y Rubio (2012), luego de la prueba de normalidad (pre y post test) se realiza un análisis de la percepción de los padres que participaron del programa, y los que no, a través 
de una prueba U de Mann Whitney. La hipótesis ad hoc es que existieron cambios significativos en la percepción de los procesos comunicacionales en los padres que participaron del programa.

\section{Discusión}

La implementación del software InCSEI en el Colegio General Gustavo Rojas Pinilla IED ha permitido, en primer lugar, visibilizar las percepciones previas y posteriores del programa, tanto para el grupo de control como para el grupo experimental.

Analizando las dinámicas de los resultados dados por la implementación de la herramienta TIC en los procesos comunicacionales de los padres con el software InCSEI, podemos inferir que las hipótesis general y específicas de la investigación y la perspectiva de la investigación está centrada en el nivel grupal (Keyton, 2017) . Esto permitió la validación de las hipótesis planteadas en la investigación bajo lo establecido en la prueba U de Mann Whitney (Berlanga y Rubio, 2012).

La implementación del programa en el grupo experimental permitió un aumento de la productividad en el uso de una comunicación asertiva. Se confirma la vinculación de las personas a las dinámicas institucionales, como lo plantean Sadia et al., (2016). Esto se reconoce en participación de los padres del grupo experimental a través de la comunicación constante con los docentes.

Con la Identificación de las necesidades de comunicación docentes - padres en la escuela, se reafirma que esto fue logrado a través del uso del Software InCSEI, como acorde a Monteserín (2014), en el que se comprueba que, al implementar un sistema propio utilizando la internet, los procesos de comunicación se hacen más efectivos.

La evaluación de la efectividad del mecanismo de comunicación institucional muestra que esta es notoria, pues se evidencia en la utilidad y la efectividad del software; además por reconocerse como un ambiente global, identificándose los roles de cada uno de los prosumidores, tal como lo describe Islas (2009).

La revisión del sistema de repositorio de información permite describirlo como amigable, y efectivo. Brinda a los padres, docentes y directivos la posibilidad de recibir la información sincrónica y hacer realimentación en forma asincrónica, tal como los proponen Sadia, Mohd, Abdul y Sanif, (2015).

El mejoramiento de la comunicación en instituciones educativas es notorio, como lo afirman Barbu y Şomăcescu (2016). Las influencias de las nuevas tecnologías redefinen el concepto de la comunicación, haciendo a la escuela más ágil. Esto se logró por el uso adecuado del Software InCSEI.

Satisfacción de uso de la aplicación del software InCSEI, el uso de la herramienta propia como lo afirma Jiménez (2017), el uso de canales permiteron trasmitir en forma clara los mensajes, facilitando que los padres sean los participantes activos. Al sentir los padres que son tenidos en cuenta, aumentan su participación al uso del software.

De acuerdo con lo anterior, se puede inferir que la implementación de herramientas TIC mejoró significativamente el gra- 


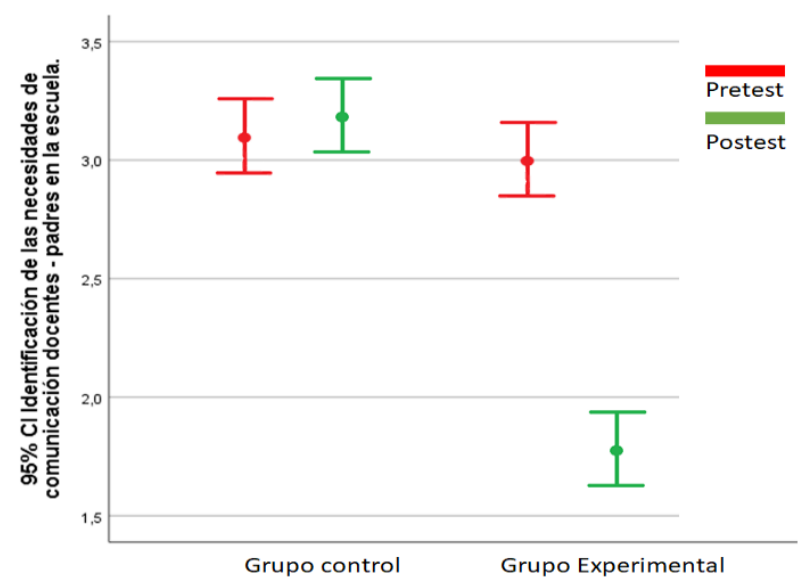

Figura 6: Identificación de las necesidades de comunicación docentes - padres en la escuela antes y después de la implementación del programa

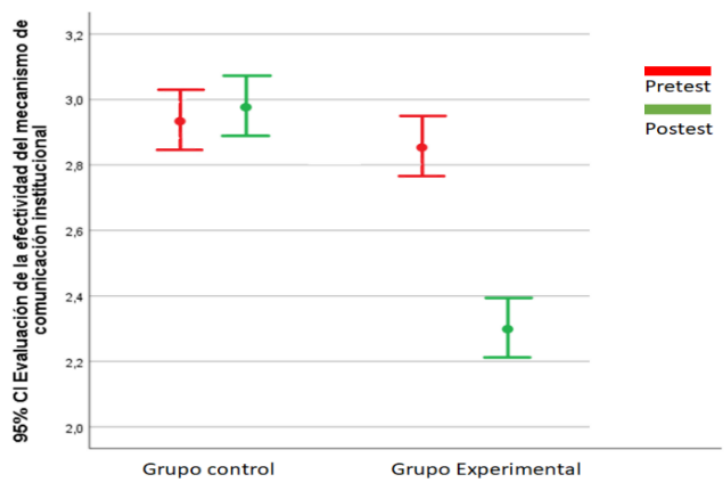

Figura 7: Evaluación de la efectividad del mecanismo de comunicación institucional antes y después de la implementación del programa 


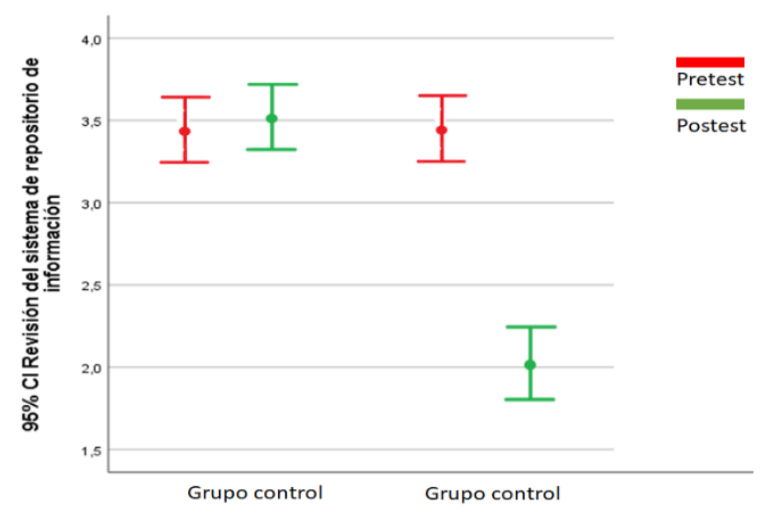

Figura 8: Evaluación de la revisión de los repositorios de información antes y después de la implementación del programa

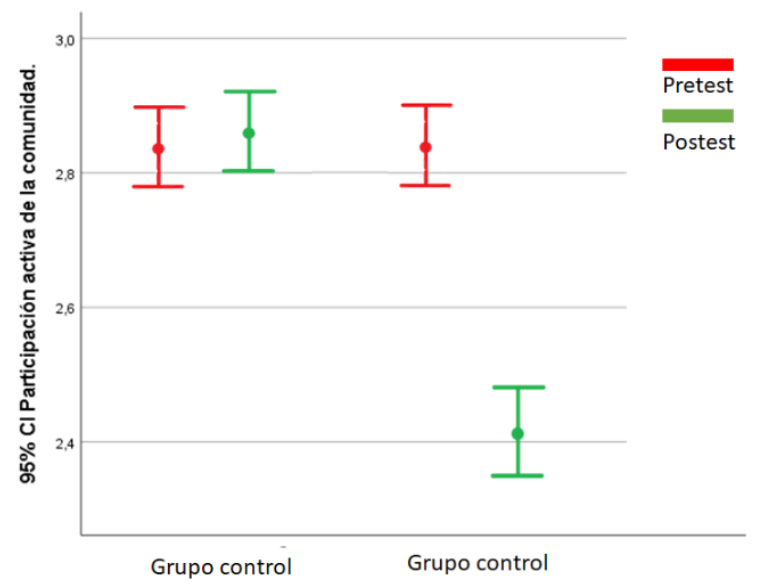

Figura 9: Evaluación de la participación de la comunidad antes y después de la implementación del programa 


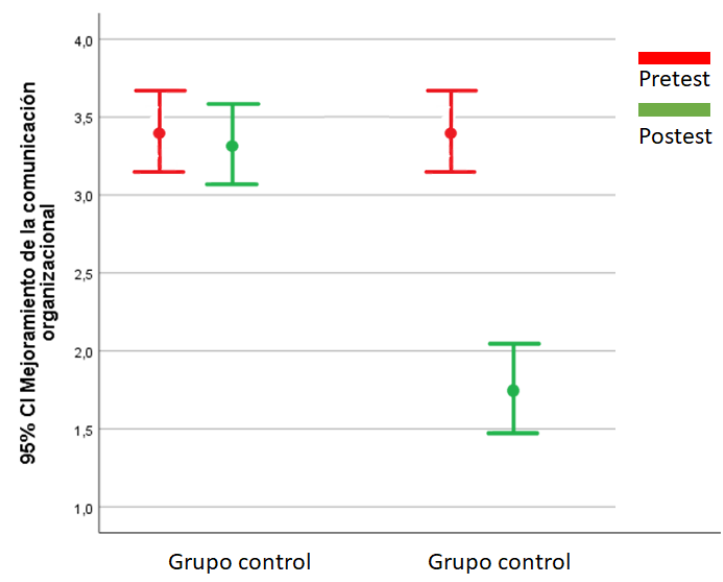

Figura 10: Evaluación de mejoramiento de la comunicación en instituciones educativas antes y después de la implementación del programa

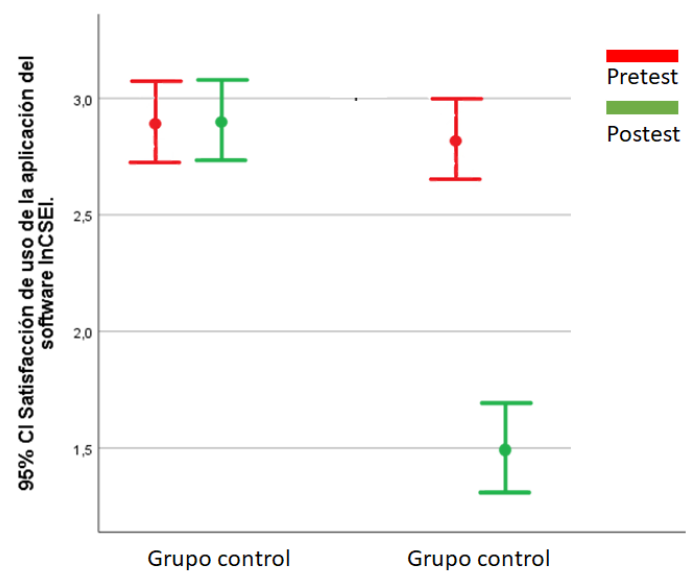

Figura 11: Evaluación de la satisfacción de uso de la aplicación del software InCSEI antes y después de la implementación del programa 
do de optimización de la comunicación en instituciones educativas, en el Colegio General Gustavo Rojas Pinilla IED de la ciudad de Bogotá D. C. en el año 2019.

El resultado obtenido se suma a lo propuesto en el paradigma de Octavio Islas, quien define los nuevos sistemas virtuales de comunicación y las acciones que se desprenden en el proceso de creación y consumo de información (Islas, 2008). Otra investigación al respecto es la de Giraldo y Maya (2016), quienes definen que la utilización de software en nuestra era mejora la comunicación de las instituciones educativas.

Además, teniendo en cuenta que en todas las dimensiones tuvo un efecto positivo, se puede también argumentar que la implementación de la herramienta TIC incrementó la efectividad tanto de los mecanismos como de los sistemas de comunicación del colegio, los cuales eran parte de las hipótesis específicas que se tenían en la investigación, logrando un avance significativo en la gestión educativa institucional (Triana y Pérez, 2016). A su vez, la utilización de herramientas TIC fomenta una cultura organizacional.

\section{Conclusiones}

La implementación del software InCSEI en el Colegio General Gustavo Rojas Pinilla IED, de la ciudad de Bogotá, se presentó como herramienta de comunicación para identificar el grado de mejora para la optimización de la comunicación. Esto se realizó desde el concepto de la Acción Comunicativa de Habermas, el cual propone la superposición al subjetivismo, con lo cual se genera una correlación con los sistemas de comunicación propuestos por Luhmann, y la utilización de las nuevas tecnologías de comunicación con el paradigma de los "prosumidores" propuesto por Octavio Islas, quien se centra en los ambientes comunicativos glocales, como Internet.

Puede concluirse que el uso de una herramienta TIC, para la comunicación institucional, optimizó satisfactoriamente el proceso de comunicación. Esto se evidencia en el alto nivel de satisfacción de los padres de familia, de la efectividad y mejoramiento del sistema, y de la disminución de la subjetividad en la comunicación antes del uso de la herramienta.

El uso de una herramienta TIC para la comunicación institucional permite el acercamiento de padres y docentes debido a la posibilidad de hacer recíproca la información sincrónica y asincrónica, ya sea de carácter académico o convivencial. Esto tiene como consecuencia que se mejore la eficacia de la gestión escolar y se aumente la participación de los padres en la vida institucional. La optimización se logró a través de la calidad y veracidad de la información que llegó a los padres de familia mediante el software InCSEI.

Es visible que la implementación de la herramienta TIC de comunicación brinda información continua entre padres y docentes, favorece la formación de los estudiantes y la comprensión de determinados, comportamientos, los cuales crean una acción comunicativa, de manera tal que se fomentan espacios de diálogo y se minimiza la subjetividad de la información.

A partir de la estrategia aplicada, se 
mejoró la agilidad de la información, y al carecer de intervenciones externas, se evidencia objetividad y eficacia. Con esta investigación se comprueba que la ecología cultural propuesta por Octavio Islas y con unos glocales adaptados a la comunicación institucional de los prosumidores.

\section{Referencias}

(1) Aguado, T. J. (2004). Introducción a las teorías de la comunicación y la información. Universidad de Murcia.

(2) Barbu, C. M. y Şomăcescu, S. M. (2016). Challenges of the Organizational Communication in the Context of the New Technologies. Journal of Advanced Research in Economics and International Business. 4(5), pp. 4-8.

Berlanga, S. V. y Rubio, H. M. (2012). Classification of nonparametric tests. How to apply them in the SPSS. Revista d'Innovació $i$ Recerca en Educació, pp. 101-113.

(3) Castro, C. L. (2015). Nivel de participación de la escuela de padres y la gestión educativa en las instituciones del municipio de Florencia Caquetá - Colombia, en el año 2015. Tesis doctoral. Universidad Norbert Wiener. Disponible en: http://repositorio.uwiener.edu. pe/handle/123456789/1443

(4) Cavazza, F. (2016). Social media landscape (2016). FredCavazza.net. Disponible en: https://fredcavazza.net/2016/04/ 23/social-media-landscape-2016/

(5) Comunicólogos. (2017). Modelo de Lasswell. Enciclopedia Comunicólogos. Disponible en: https://www.comunicologos.com/ teorias/modelo-de-lasswell/

(6) Espinoza, M. C. (2014). Metodología de investigación tecnológica: Pensando en sistemas. Soluciones Gráficas S.A.C.

(7) Fonseca, Y. M., Correa, P. A., Pineda, R. M. y Lemus, H. F. (2011). Comunicación Oral, Fundamentos y Práctica Estratégica. Pearson Educación.

(8) Garza, A. V. (2017). Los medios sociales en la comunicación de la ciencia y el ambiente. Cultura Ciencia y Tecnológia. CULCyT . 61, pp. 5-13.

(9) George, D. y Mallery, P. (2016). IBM SPSS statistics 23 step by step: A simple guide and reference. Routledge.

(10) Giraldo, D. A. y Maya, F. C. (2016). Modelos de ecología de la comunicación: análisis del ecosistema comunicativo. Palabra Clave. 19 (3), pp. 746-768.

(11) Habermas, J. (1981). Racionalidad de la acción. Teoría de la acción comunicativa, Volúmenes 1-2. Editorial Trotta.

(12) Hasbún, S. H. (2015). Las TIC y el Nuevo Paradigma de la Comunicación Institucional. Políticas y Es- 
trategias en la Era de la Convergencia. Tesis doctoral. UNED. Disponible en: https://dialnet.unirioja. es/servlet/tesis? codigo $=67424$

(13) Hernández, S. R., Fernández, C. C. y Baptista, L. P. (2014). Metodología de la investigación. McGrawHill Interamericana.

(14) Islas, O. (2008). La sociedad de la ubicuidad, los prosumidores y un modelo de comunicación para comprender la complejidad de las comunicaciones digitales. Revista Latinoamericana de Ciencias de la Comunicación. 7, pp. 68-77. Disponible en: https://www.redalyc.org/ articulo.oa?id=199520724016

(15) Islas, O. (2009). La convergencia cultural a través de la ecología de medios. Comunicar: Revista Cientifica Iberoamericana de Comunicación y Educación. 33, pp. 2533.

(16) Jiménez, S. L. (2017). Módulo de intervención para abrir canales de comunicación asertiva entre docentes, padres de familia y estudiantes de secundaria y media. Tecnológico de Monterrey. Disponible en: ://repositorio.tec.mx/ bitstream/handle/11285/622459/

Tesis\%20JSLM.pdf?sequence $=9 \&$ amp;isAllowed $=\mathrm{y}$

(17) Keyton, J. (2017). Communication in Organizations. Annual Review of Organizational Psychology and Organizational Behavior. 4, pp. 501526.
(18) Lasswell, H. D. (1948). The structure and function of communication in society. The communication of ideas. The Institute for Religious and Social Studies.

(19) Martinez-Alvarez E., Rosa C.M.E., Yangali V. J.S. (2021) Integration of ICT in academic and administrative management processes. PSYCHOLOGY AND EDUCATION (2021) 58(1): 5594-5599. http://psychologyandeducation. net/pae/index.php/pae/article/ view/2179/1899

(20) Monteserín, L. P. (2014). Comunicación institucional y social media en Castilla y León: análisis y elaboración de un plan estratégico de comunicación 2.0. Tesis doctoral. Universidad de Burgos. Disponible en: https://dialnet.unirioja. es/servlet/tesis? codigo $=44285$

(21) Niño, R. V. (2011). Metodología de la Investigación: diseño y ejecución. Ediciones de la U.

(22) Papic, D. G. (2016). La comunicación organizacional en entidades educativas. Tesis doctoral. Universidad de Málaga, departamento de Comunicación Audiovisual y $\mathrm{Pu}-$ blicidad. Disponible en: http://hdl. handle.net/10630/12060

(23) Pignuoli, O. S. (2013). El Modelo Sintético de Comunicación de Niklas Luhmann. Cinta moebio. 47, pp. 59-73. Disponible en: http://dx.doi.org/10.4067/S0717554X2013000200001 
(24) Sadia, A., Mohd, B., Abdul, K. Z y Sanif, S. (2016). The Relationship between Organizational Communication and Employees Productivity with New Dimensions of Effective Communication Flow. Journal of Business and Social Review in Emerging Economies. 2(2), pp. 93100. Disponible en: https://doi.org/ 10.26710/jbsee.v2i2.35

(25) Triana, M. M. y Pérez, A. N. (2016). Fortalecimiento de la comunicación organizacional entre docentes y directivos docentes, a partir de círculos de calidad como herramienta de gestión directiva en el Colegio Brasilia Bosa I.E.D. sede a jornada mañana. Universidad Libre. Disponible en: http://hdl.handle.net/ 10901/9733

(26) UNESCO (2019). Lanzamiento de la Alianza Global de Plataformas de Comunicación Académica de Acceso Abierto para democratizar el conocimiento. Disponible en: https:// en.unesco.org/news/launch-globalalliance-open-access-scholarlycommunication-platformsdemocratize-knowledge13/05/2020

(27) Yangali V. J.S.; Arboleda O. J.A.; Arispe Albuqueque, C. M. (2021) Revista Ibérica de Sistemas e Tecnologias de Informação; Lousada Iss. E39, (156-169. https: //www.scopus.com/record/display. uri?eid $=2-\mathrm{s} 2.0-85101910835 \&$ origin $=$ inward $\&$ txGid $=$ 4ace34c5845ce60b3d67d2f56cc13286 